\title{
Stability of a Noncanonical Scalar Field Model during Cosmological Date
}

\author{
Z. Ossoulian, ${ }^{1}$ T. Golanbari, ${ }^{1}$ H. Sheikhahmadi, ${ }^{1,2}$ and Kh. Saaidi ${ }^{1}$ \\ ${ }^{1}$ Department of Physics, Faculty of Science, University of Kurdistan, Pasdaran Street, Sanandaj 66177-15175, Iran \\ ${ }^{2}$ Institute for Advance Studies in Basic Sciences (IASBS), Gava Zang, Zanjan 45137-66731, Iran \\ Correspondence should be addressed to Z. Ossoulian; zossoulian@gmail.com
}

Received 25 July 2015; Revised 19 November 2015; Accepted 23 November 2015

Academic Editor: Sally Seidel

Copyright (C) 2016 Z. Ossoulian et al. This is an open access article distributed under the Creative Commons Attribution License, which permits unrestricted use, distribution, and reproduction in any medium, provided the original work is properly cited. The publication of this article was funded by SCOAP S $^{3}$

Using the noncanonical model of scalar field, the cosmological consequences of a pervasive, self-interacting, homogeneous, and rolling scalar field are studied. In this model, the scalar field potential is "nonlinear" and decreases in magnitude with increasing the value of the scalar field. A special solution of the nonlinear field equations of $\phi$ that has time dependency as fixed point is obtained. The fixed point relies on the noncanonical term of action and $\gamma$-parameter; this parameter appeared in energy density of scalar field redshift. By means of such fixed point the different eigenvalues of the equation of motion will be obtained. In different epochs in the evolution of the Universe for different values of $q$ and $n$, the potentials as a function of scalar field are attained. The behavior of baryonic perturbations in linear perturbation scenario as a considerable amount of energy density of scalar field at low redshifts prevents the growth of perturbations in the ordinary matter fluid. The energy density in the scalar field is not appreciably perturbed by nonrelativistic gravitational fields, in either the radiation or matter dominant or scalar field dominated epoch.

\section{Introduction}

During the two past decades, undoubtedly people believe the Universe is undergoing an accelerated expansion phase. This interesting and unanticipated result comes from some observations including Cosmic Microwave Background (CMB) $[1,2]$, Supernovae type Ia (SNeIa) [3, 4], Baryonic Acoustic Oscillations (BAO) [5, 6], Observational Hubble Data (OHD) $[7,8]$, Sloan Digital Sky Survey (SDSS) [9, 10], and Wilkinson Microwave Anisotropy Probe (WMAP) [11, 12]. To justify such accelerated epoch, people mostly consider an unknown form of matter which produces a negative pressure, namely, dark energy [13]. These observations also anticipate that the nonluminous components of the Universe (dark energy $68.3 \%$, dark matter $26.6 \%[14,15]$ ) are $94.9 \%$ and ordinary matter (baryonic and radiation) is only $5.1 \%$. It is notable that, studying the origin and nature of dark energy, the best candidate is cosmological constant, c.c., $\Lambda$ [16-21]. But unfortunately this interesting attractive candidate of dark energy suffers two well-known problems, fine tuning and coincidence problems. The former moot point refers to the difference between the theoretical anticipation of the energy density of $\Lambda$ and what is risen from observations. The latter comes back to the following question: why the ratio of matter and dark energy densities in present epoch is so close to unit? [22]. These two problems convince scientists to make other proposals to investigate the behaviour of dark energy. Accordingly people are interested in searching for some proposals and models which resolve the two mentioned problems and also have a strong support from both theoretical and experimental point of views. People unusually have proposed different proposals based on two completely different perspectives; one of them is a geometrical scenario and the other is related to the manipulation the matter components of the Universe. For the former model one can mention $f(R)$ [23-26], $f(T)$ [27-31], and $f(G)$ [32-35] gravities and modification or combination of them based on cosmological purpose, and for the latter, for example, we can name some models which have arisen from quantum gravity (e.g., holographic model) [36-40], space time fluctuations (e.g., age and new-age graphic models) [41-44], and vacuum quantum fluctuations (e.g., Casimir 
effect in large scale) [41, 45-47] and also we are able to allude to the scalar field proposals. It is well known that scalar field scenario usually is considered as the first and best candidate for new phenomena which appeared in physics, for instance, Yukawa model of strong force [48, 49], LandauGinzburg mechanism [50], Higgs mechanism [51, 52], and inflation [53-56]. Also it is notable that scalar fields under the general coordinate reparametrization have the simplest behavior, which has attracted more attention recently. It is considerable as a special category; we can split the scalar field scenarios to canonical and noncanonical models. For the canonical proposal one can mention Brans-Dicke $[57,58]$, quintessence [59-62], phantom [63-66], quintom [67-69], and chameleon [70-78]. Also for noncanonical mechanism we can refer to k-essence [79-81], Dirac-Born-Infeld model [82], tachyon [83], and so on. It should be stressed that the noncanonical mechanism has some advantages against canonical mechanism. For instance, although quintessence proposal as a tracker model resolves coincidence problem, it needs to be highly tuned; it is notable that in noncanonical scenario both coincidence and fine tuning problems could be resolved. Another interesting aspect of the noncanonical model (k-essence) against quintessence is that the kinetic term in such scenario can source the c.c. These interesting aspects of noncanonical model motivated us to investigate the stability of this scenario in different epochs in cosmology. For this aim we are actually interested in the behavior, in linear perturbation theory, of density perturbations in the scalar field energy density and in the densities of baryons and radiation. It is obvious that scalar field energy density fluctuations tend to decay inside of the horizon, while in a baryon dominated universe the behavior of baryonic perturbations is effectually the same as in the canonical scenario. On the other hand, if the energy density in the homogeneous part of the scalar field is considerable, ordinary matter perturbations cannot grow. Since scalar field perturbations do not grow inside the horizon, the scalar field energy density will remain very much smoother than the baryonic, so the peaks in the matter distribution will be almost entirely baryonic. The mass of the scalar field fluctuation (second derivative of the scalar potential) is related to the horizon size; the scalar field fluctuation is extremely light. If the scalar field were to dominate early enough, it would suppress growth of baryonic structure on small scales. This is because the Universe expands so fast that the perturbations cannot collapse. Note that the scalar field has to be minimally coupled to matter or must be very weakly coupled to ordinary matter so that it does not drag the matter perturbations with it and thereby prevent them from collapsing, even before the scalar field comes to dominate the energy density of the Universe. This is one reason why we have to suppose that the Universe has only recently become dominated by the scalar field energy density, if galaxies formed by gravitational instability. This effect motivates the choice of potentials $V(\phi)$ discussed below. We study a class model of the potential of the scalar field. In this model, we assume that the energy density of the scalar field redshifts in a certain way and then determines the potential of the scalar field that is required. We find out that the scalar field potential is "nonlinear" which typically tends to be made from negative powers of the scalar field. We have not succeeded in determining the general solution of the scalar field equation of motion, but a special solution can be found (in which the scalar field energy density redshifts in the requisite manner). Somewhat remarkably, we find that this solution dominates at large time and a study of phase space shows that it is an attractive and time dependent fixed point (in the cases of interest, it is the only attractive fixed point in phase space). This solution may, therefore, be chosen as a background solution for a study of the evolution of density inhomogeneities in linear perturbation theory.

The scheme of this paper is as follows. In Section 1 some aspects of both canonical and noncanonical mechanisms, and, also, the importance of scalar field proposal beside the motivations of this work were discussed as introduction. In Section 2 the general framework of this work including the math calculations and also the equations of energy density and pressure of noncanonical model will be discussed; also we will obtain the general form of the potential for this model. In Section 3, by means of a perturbation mechanism, we will solve the equation of motion of scalar field. To solve this equation we should find out the fixed point of the model, and by using it the eigenvalues of the equation will be obtained; based on this values we will discuss different conditions to investigate the stability or nonstability of the model. In Section 4 we will consider different epochs in the evolution of the Universe, namely, radiation, matter, and scalar field dominans, respectively. And, at last, results and conclusions of the work will be brought in Section 5 .

\section{The Model}

Using canonical model of scalar field, the energy density of potential, time derivative of scalar field, the ratio of the energy density of scalar field to radiation and matter, and also the wave equation of scalar field have been studied. In phase space the values of special solution and general solution of wave equation have been obtained and the critical points and perturbations around these critical points in phase space equations have been investigated. Specifying the value of $q$ with respect to the ratio of energy density of scalar field to the radiation and matter, the dominance of the energy density of scalar field at present time and the positive acceleration of the Universe at current epoch have been explained. In this paper we want to study the above-mentioned conditions by means of noncanonical scalar field to get better results in comparison to the results which were obtained in ordinary canonical proposal. It is considerable that we consider homogeneous, isotropic, and spatially flat space time which is given by the line element:

$$
d s^{2}=-d t^{2}+a^{2}\left(d r^{2}+r^{2} d \theta^{2}+r^{2} \sin ^{2} \theta d \phi^{2}\right) .
$$

The noncanonical version of Einstein scalar field action is defined as $[79,80]$

$$
\mathcal{S}=\int \sqrt{-g} d^{4} x\left(\frac{M_{p}^{2}}{2} R+F(X)-V(\phi)+\mathscr{L}_{m}\right),
$$


where $g$ is the determinant of the metric $g_{\mu \nu}, R$ is the Ricci scalar, and also $M_{p}$ is the Planck mass. Potential of scalar field is denoted by $V(\phi), \mathscr{L}_{m}$ is introduced as ordinary matter Lagrangian, and the kinetic term of noncanonical scalar field is expressed by an arbitrary function $F(X)$, in which $X=$ $-\left(g^{\mu \nu} \nabla_{\mu} \phi \nabla_{\nu} \phi\right) / 2$. It should be stressed that the noncanonical scalar field Lagrangian in question is

$$
\mathscr{L}_{\phi}=F(X)-V(\phi) \text {. }
$$

Variation of action (2) with respect to $g^{\mu \nu}$ leads to

$$
G_{\mu \nu}=R_{\mu \nu}-\frac{1}{2} g_{\mu \nu} R=\frac{1}{M_{p}^{2}}\left(T_{\mu \nu}^{m}+T_{\mu \nu}^{\phi}\right) .
$$

By varying (2) with respect to $\phi$ and considering (1), one has

$$
\left(2 X F_{X X}+F_{X}\right) \ddot{\phi}+3 F_{X} H \dot{\phi}+\widetilde{V}(\phi)=0,
$$

where overdot denotes a derivative with respect to cosmic time and tilde indicates derivative with respect to $\phi$. Here $R_{\mu \nu}$ is the Ricci tensor, $F_{X}=d F(X) / d X$, and $F_{X X}=d^{2} F(X) / d X^{2}$. For an arbitrary Lagrangian, $\mathscr{L}_{m}$, the stress-energy tensor is defined as

$$
T_{\mu \nu}=\frac{-2}{\sqrt{-g}} \frac{\delta\left(\sqrt{-g} \mathscr{L}_{m}\right)}{\delta g^{\mu \nu}}
$$

Therefore stress-energy tensor of scalar field, $T_{\mu \nu}^{\phi}$, is given by

$$
T_{\mu \nu}^{\phi}=g_{\mu \nu}[F(X)-V(\phi)]+\left(\nabla_{\mu} \phi \nabla_{\nu} \phi\right) F_{X},
$$

and $T_{\mu \nu}^{m}$ is the stress-energy tensor of ordinary matter such that for a perfect fluid cosmological matter one has

$$
T_{\mu \nu}=(\rho+p) U_{\mu} U_{\nu}+p g_{\mu \nu} .
$$

Here $U^{\mu}$ is four-vector of a fluid. From (1) we have

$$
T_{\mu}^{\nu}=\operatorname{diag}(-\rho, p, p, p) .
$$

Here $\rho=\rho_{r}+\rho_{m}$ and $p=p_{r}+p_{m}$, where subscripts $r$ and $m$ refer to energy density of radiation and energy density of baryonic and cold dark matter, respectively. Utilizing the usual definition of the stress-energy tensor, (7) and (9), the energy density and the pressure of scalar field are defined as

$$
\begin{aligned}
& \rho_{\phi}=T_{00}^{(\phi)}=2 X F_{X}-F(X)+V(\phi), \\
& p_{\phi}=T_{i i}^{(\phi)}=F(X)-V(\phi) .
\end{aligned}
$$

By omitting $\rho_{r}$ and $\rho_{m}$ and also by means of (4), (7), (9), and (1), one can obtain

$$
\begin{aligned}
H^{2} & =\frac{8 \pi}{3 M_{p}^{2}}\left(2 X F_{X}-F(X)+V(\phi)\right), \\
\frac{\ddot{a}}{a} & =H^{2}+\dot{H}=\frac{-1}{3 M_{p}^{2}}\left(F+X F_{X}-V(\phi)\right) .
\end{aligned}
$$

For regarding the spatial homogeneity of cosmology, we suppose that the scalar field is just a function of time so $X=\dot{\phi}^{2} / 2$. By considering (10a) and (10b) time derivative of scalar field and the related potential are reduced to

$$
\begin{aligned}
\dot{\phi}^{2} & =\frac{\rho_{\phi}+p_{\phi}}{F_{X}}, \\
V(\phi) & =\frac{\left(\rho_{\phi}-p_{\phi}\right)}{2}+F(X)-X F_{X} .
\end{aligned}
$$

We suppose that there was not any interaction between components of fluid, so that conservation equation of stressenergy equation is satisfied for all fluid components of the Universe as

$$
\dot{\rho}_{i}+3 H\left(\rho_{i}+p_{i}\right)=0
$$

Here $\rho_{i}=\rho_{r}, \rho_{m}, \rho_{\phi}$. In addition, we assume that the scalar field energy density redshifts are given by

$$
\rho_{\phi}=\rho_{\phi}^{(0)}\left(\frac{a_{0}}{a}\right)^{q}
$$

We assume $q \neq 0$. Therefore by means of (14) and (15) one gets

$$
p_{\phi}=\left(\frac{q-3}{3}\right) \rho_{\phi}
$$

where $\omega=(q-3) / 3$ is equation of state parameter of scalar field. By considering $F(X)=F_{0} X^{n}$ and using (13) and (15) one receives

$$
\begin{gathered}
\dot{\phi}=\left[\frac{\rho_{\phi}^{(0)} q 2^{n-1}}{3 F_{0} n}\right]^{1 / 2 n}\left(\frac{a_{0}}{a}\right)^{q / 2 n}, \\
V(\phi)=\left[\frac{6 n-(2 n-1) q}{6 n}\right] \rho_{\phi}^{(0)}\left(\frac{a_{0}}{a}\right)^{q} .
\end{gathered}
$$

It should be noted that, for $n=1 / 2$, the coefficient $\ddot{\phi}$ in (5) is equal to zero; therefore we will not consider this case.

\section{A Component Dominated Cosmology}

As we mentioned in previous sections, we have assumed that the components of the Universe could be considered as radiation, matter (ordinary and cold dark matter), and scalar field. But in this section we will suppose that the contribution of the Universe components in the stress-energy tensor is only one of them. In fact we assume that in any epoch only the energy density of related component is dominated and therefore other components of perfect fluid could be neglected. According to conservation equation for energy density, (14), and the related equation of state, $p=(\gamma / 3-1) \rho$, the energy density of the dominant component is obtained as

$$
\rho_{\gamma}=\rho_{\gamma}^{(0)}\left(\frac{a_{0}}{a}\right)^{\gamma}
$$


where $\rho_{\gamma}^{(0)}$ is the value of energy density at $a=a_{0}$ and $(\gamma / 3-1)$ is the equation of state parameter of dominant component. So the Friedman equation (11) could be written as follows:

$$
\left(\frac{\dot{a}}{a}\right)^{2}=\frac{\rho_{\gamma}^{(0)}}{3 M_{p}^{2}}\left(\frac{a_{0}}{a}\right)^{\gamma} .
$$

Using (17) and (20) one obtains

$$
\frac{\dot{\phi}}{\dot{a}}=\left(\frac{q \rho_{\phi}^{(0)} 2^{n-1}}{3 F_{0} n}\right)^{1 / 2 n}\left(\frac{3 M_{p}^{2}}{\rho_{\gamma}^{(0)} a_{0}^{2}}\right)^{1 / 2}\left(\frac{a}{a_{0}}\right)^{(\gamma n-q-2 n) / 2 n} .
$$

Hence, by integration the above relation, the scalar field results as

$$
\begin{aligned}
\phi-\phi_{0}= & \frac{2 n}{\gamma n-q}\left(\frac{q \rho_{\phi}^{(0)} 2^{n-1}}{3 F_{0} n}\right)^{1 / 2 n}\left(\frac{3 M_{p}^{2}}{\rho_{\gamma}^{(0)}}\right)^{1 / 2} \\
& \cdot\left(\frac{a}{a_{0}}\right)^{(\gamma n-q) / 2 n},
\end{aligned}
$$

where $\phi_{0}$ is the value of $\phi$ at $a=a_{0}$. And also, from (18) and (22), we have

$$
V(\phi)=V_{0}\left(\phi-\phi_{0}\right)^{2 n q /(q-\gamma n)},
$$

where

$$
\begin{aligned}
V_{0} & =\frac{[6 n-(2 n-1) q] \rho_{\phi}^{(0)}}{6 n}\left[\frac{2 n}{\gamma n-q}\left(\frac{q \rho_{\phi}^{(0)} 2^{n-1}}{3 F_{0} n}\right)^{1 / 2 n}\right. \\
& \left.\cdot\left(\frac{3 M_{p}^{2}}{\rho_{\gamma}^{(0)}}\right)^{1 / 2}\right]^{2 n q /(q-\gamma n)} \cdot
\end{aligned}
$$

The Einstein scalar field equations (for such potential) have a special solution. In this section we study spatially homogeneous perturbations about this special solution. To do this we should study the structure of the four-dimensional, spatially homogeneous, phase space $(\phi, \dot{\phi})$. Therefore, we consider the scalar field equation of motion, which is given by

$$
\begin{aligned}
y^{\prime \prime} & +\left(\frac{4(n+1)-\gamma(2 n-1)}{2(2 n-1)}\right) \frac{y^{\prime}}{x} \\
& +\frac{q V_{0} 2^{n}}{F_{0}(2 n-1)(q-\gamma n)}\left(\frac{3 M_{p}^{2}}{\rho_{\gamma}^{(0)}}\right)^{n} x^{n(\gamma-2)} \\
& \cdot \frac{y^{((2 n-1) q+\gamma n) /(q-\gamma n)}}{\left(y^{\prime}\right)^{2 n-2}}=0,
\end{aligned}
$$

where prime indicates derivative with respect to $x$ and we have changed the variables as $x=a / a_{0}$ and $y=\phi-\phi_{0}$. A special solution of differential equation (25) is introduced as

$$
y_{e}=k x^{\alpha}
$$

and, by substituting (26) into (25), $k$ and $\alpha$ are obtained as

$$
\begin{aligned}
& k=\frac{2 n}{\gamma n-q}\left[\frac{q \rho_{\gamma}^{(0)} 2^{n-1}}{3 F_{0} n}\right]^{1 / 2 n}\left(\frac{3 M_{p}^{2}}{\rho_{\gamma}^{(0)}}\right)^{1 / 2}, \\
& \alpha=\frac{\gamma n-q}{2 n} .
\end{aligned}
$$

To study the structure of the phase space of scalar fields, one can make a change of variable as

$$
y(x)=y_{e}(x) u(x) .
$$

In above redefinition $y_{e}(x)$, the special solution of differential equation is unperturbed part of scalar field and $u(x)$ is the perturbed part of the scalar field which should have a stable and attractor property. So, for stability of the solutions of scalar field, differential equation (25) should solve for the perturbed part. It is notable that all dynamical information related to equation of motion are at evaluation equation $u(x)$. By substituting the general solution into differential equation (25) the result is as follows:

$$
\begin{aligned}
& u^{\prime \prime}+\left[\frac{2(n+1)}{(2 n-1)}-\frac{\gamma}{2}+2 \alpha\right] \frac{u^{\prime}}{x} \\
& +\left[\frac{3}{(2 n-1)}-\frac{\gamma}{2}+\alpha\right]\left[u-\frac{u^{(q(2 n-1)+\gamma n) /(q-\gamma n)}}{\left((x / \alpha) u^{\prime}+u\right)^{2 n-2}}\right] \frac{\alpha}{x^{2}} \\
& =0 .
\end{aligned}
$$

By changing the variable $x=e^{t}$ and rewriting (29) in terms of $(u(t), t)$, one has

$$
\begin{aligned}
\ddot{u}+ & {\left[\frac{3}{(2 n-1)}-\frac{\gamma}{2}+2 \alpha\right] \dot{u} } \\
& +\alpha\left[\frac{3}{(2 n-1)}-\frac{\gamma}{2}+\alpha\right]\left[u-\frac{u^{(q(2 n-1)+\gamma n) /(q-\gamma n)}}{(\dot{u} / \alpha+u)^{2 n-2}}\right] \\
& =0
\end{aligned}
$$

where as formerly overdot indicates derivative with respect to $t$. The critical point of this system is defined as a point at which the velocities could be neglected. Therefore, the only critical point is obtained as $\dot{u}=0$. Hence according to (25) we are able to study the evolution of $u(t)$. To do this we use phase space to explain the stability of this nonlinear problem by means of linear analysis. Therefore, in phase space $(u(t), \dot{u}(t)),(30)$ could be rewritten as

$$
\begin{aligned}
\dot{u}(t) & =p(t) \\
\dot{p}(t) & =-\alpha\left[\frac{3}{(2 n-1)}-\frac{\gamma}{2}+\alpha\right] \\
\cdot & {\left[u(t)-\frac{u(t)^{((2 n-1) q+\gamma n) /(q-\gamma n)}}{(p(t) / \alpha+u(t))^{2 n-2}}\right] } \\
- & {\left[\frac{3}{(2 n-1)}-\frac{\gamma}{2}+2 \alpha\right] p(t) . }
\end{aligned}
$$


Then, for $p(t)=0$, we have

$$
u(t)^{2 n-1}-u(t)^{(q(2 n-1)+\gamma n) /(q-\gamma n)}=0 .
$$

The point $\left(u_{0}, p_{0}\right)=(1,0)$ is the only acceptable critical point for (31). The system at critical point does not have evolution and so the differential equation, (25), just has the special solution, (26). Of course, at $p(t)=0$, there are a number of critical points, such that $u(t)$ is $\left[(\gamma n-q) 8^{-1} n^{-2}\right]$ the root of unity. $u(t)$ should be real, so we do not work with the complex fixed points. It is seen that, by changing $y(x) \rightarrow-y(x)$, the differential equation (25), for some values of $q$ and $n$, is invariant. In this case there are two critical points, but our purpose is to explain the solutions in which $y^{\prime}(x)>0$. So one of the critical points is omitted. In the second part of function $y(x)=y_{e}(x) u(x), u(x)$ can be perturbed by adding $u_{1}(t)$ to the critical point $\left(u_{0}, p_{0}\right)$ and substituting at phase space equations (31). The result is (in fact $u_{1}(t)$ is a small fluctuation around the critical point $\left.\left(u_{0}, p_{0}\right)=(1,0)\right)$ added and it is transformed into the critical point $\left(u_{0}+u_{1}(t), p_{0}+p_{1}(t)\right)=$ $\left(1+u_{1}(t), p_{1}(t)\right)$

$$
\begin{aligned}
& \dot{u}_{1}(t)=0+p_{1}(t) \\
& \dot{p}_{1}(t)=\gamma\left[\frac{q}{2}-\frac{3 n}{2 n-1}\right] u_{1}(t)+\left[q-3-\frac{\gamma}{2}\right] p_{1}(t) .
\end{aligned}
$$

The eigenvalues of small oscillations around critical point are given by

$$
\lambda_{n, q, \gamma}=f(n, q, \gamma) \pm i g(n, q, \gamma)
$$

where

$$
\begin{aligned}
& f(n, q, \gamma)=\frac{2 q-6-\gamma}{4} \\
& g(n, q, \gamma)=\left[\gamma\left(\frac{3 n}{2 n-1}-\frac{q}{2}\right)-\frac{(q-3-\gamma / 2)^{2}}{4}\right]^{1 / 2} .
\end{aligned}
$$

The eigenvalues $\lambda$ for different $n$ and $q$ will be evaluated.

\section{Investigation of Epochs Dominant}

In this section we want to examine our results of previous section for different epochs during evolution of the Universe.

4.1. Radiation Dominated Epoch of Cosmology. We assume the radiation dominant era, and therefore one can neglect the other components of perfect fluid in the Universe. According to conservation equation of radiation, (14), and equation of state for radiation, $p=\rho / 3$, one can observe that in radiation epoch $\gamma=4$. Thus the radiation energy density resulted as

$$
\rho_{r}=\rho_{r}^{(0)}\left(\frac{a_{0}}{a}\right)^{4}
$$

where $\rho_{r}^{(0)}$ is the value of radiation energy density at $a=a_{0}$. So, according to (22), (23), and (24), we have

$$
\begin{aligned}
\phi-\phi_{0}= & \frac{2 n}{4 n-q}\left(\frac{q \rho_{\phi}^{(0)} 2^{n-1}}{3 F_{0} n}\right)^{1 / 2 n}\left(\frac{3 M_{p}^{2}}{\rho_{r}^{(0)}}\right)^{1 / 2} \\
& \cdot\left(\frac{a}{a_{0}}\right)^{(4 n-q) / 2 n},
\end{aligned}
$$

and for the potential one has

$$
V(\phi)=V_{0}\left(\phi-\phi_{0}\right)^{2 n q /(q-4 n)},
$$

where

$$
\begin{aligned}
V_{0} & =\frac{[6 n-(2 n-1) q] \rho_{\phi}^{(0)}}{6 n}\left[\frac{2 n}{4 n-q}\left(\frac{q \rho_{\phi}^{(0)} 2^{n-1}}{3 F_{0} n}\right)^{1 / 2 n}\right. \\
& \left.\cdot\left(\frac{3 M_{p}^{2}}{\rho_{r}^{(0)}}\right)^{1 / 2}\right]^{2 n q /(q-4 n)} .
\end{aligned}
$$

The Einstein scalar field equations (5) (with this potential) have a special solution. So to study spatially homogeneous perturbations about this special solution, we need to linearize the scalar field equation of motion. Therefore, according to (33), the linearized equation in this epoch is given as

$$
\begin{aligned}
& \dot{u}_{1}(t)=0+p_{1}(t) \\
& \dot{p}_{1}(t)=4\left[\frac{q}{2}-\frac{3 n}{2 n-1}\right] u_{1}(t)+(q-5) p_{1}(t) .
\end{aligned}
$$

The eigenvalues of small oscillations around critical point are given by

$$
\lambda_{n, q}=f(n, q) \pm i g(n, q)
$$

where

$$
\begin{aligned}
& f(n, q)=-\frac{(5-q)}{2}, \\
& g(n, q)=\left[\frac{48 n-\left(25-2 q+q^{2}\right)(2 n-1)}{4(2 n-1)}\right]^{1 / 2} .
\end{aligned}
$$

These eigenvalues for $0<q<3$ and different $n$ are given at Table 1 (as was mentioned before, for $0<q<3$, the ratio of scalar field density to radiation density is an increasing function of time). It is shown that (from Table 1 ) for all values of $n=1,1.5,2$ and $q=1,2,2.5$ the eigenvalues $\lambda$ are $\lambda=\alpha \pm i \beta$ where $\alpha<0$ and $\beta \neq 0$; then all critical points are spiral and stable.

4.2. Matter Dominated Epoch. We assume the matter is dominated and therefore other components of the Universe could be omitted. According to conservation equation of 
TABLE 1: The eigenvalues $\lambda$ for different values of $n$ and $q$, in radiation dominated eppoch.

\begin{tabular}{cccc}
\hline & & $\lambda$ & $V(\phi) \sim$ \\
\hline$n=1$ & $q=1$ & $-2 \pm 2.4 i$ & $\left(\phi-\phi_{0}\right)^{-0.7}$ \\
& $q=2$ & $-1.5 \pm 1.2 i$ & $\left(\phi-\phi_{0}\right)^{-2}$ \\
& $q=2.5$ & $-1.2 \pm 2.3 i$ & $\left(\phi-\phi_{0}\right)^{-3.3}$ \\
\hline \multirow{2}{*}{$n=\frac{3}{2}$} & $q=1$ & $-2 \pm 1.7 i$ & $\left(\phi-\phi_{0}\right)^{-0.6}$ \\
& $q=2$ & $-1.5 \pm 1.7 i$ & $\left(\phi-\phi_{0}\right)^{-1.5}$ \\
& $q=2.5$ & $-1.3 \pm 1.6 i$ & $\left(\phi-\phi_{0}\right)^{-2.1}$ \\
\hline \multirow{3}{*}{$n=2$} & $q=1$ & $-2 \pm 1.4 i$ & $\left(\phi-\phi_{0}\right)^{-0.6}$ \\
& $q=2$ & $-1.5 \pm 1.3 i$ & $\left(\phi-\phi_{0}\right)^{-1.3}$ \\
& $q=2.5$ & $-1.2 \pm 1.2 i$ & $\left(\phi-\phi_{0}\right)^{-1.8}$ \\
\hline
\end{tabular}

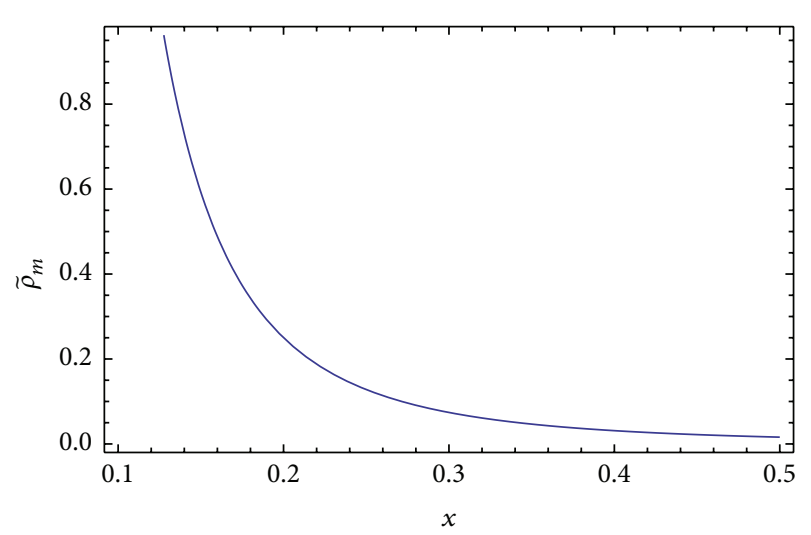

FIGURE 1: $\widetilde{\rho}_{m}:=\rho_{m} / \rho_{m}^{(0)}$ versus $x$ is plotted.

matter, (14), and the equation of state for this era, one can find that $\gamma=3$; therefore the energy density of matter resulted as

$$
\rho_{m}=\rho_{m}^{(0)}\left(\frac{a_{0}}{a}\right)^{3}
$$

where $\rho_{m}^{(0)}$ is the value of energy density of matter at $a=a_{0}$ (see Figure 1).

So one can find that

$$
\begin{aligned}
\phi-\phi_{0}= & \frac{2 n}{3 n-q}\left[\frac{q \rho_{\phi}^{(0)} 2^{n-1}}{3 F_{0} n}\right]^{1 / 2 n}\left(\frac{3 M_{p}^{2}}{\rho_{m}^{(0)}}\right)^{1 / 2} \\
& \cdot\left(\frac{a}{a_{0}}\right)^{(3 n-q) / 2 n},
\end{aligned}
$$

and $\phi_{0}$ is the value of $\phi$ at $a=a_{0}$, and the potential comes to the following form:

$$
V(\phi)=V_{0}\left(\phi-\phi_{0}\right)^{2 n q /(q-3 n)}
$$

TABLE 2: The eigenvalues $\lambda$ for different values of $n$ and $q$, in matter dominated epoch.

\begin{tabular}{cccc}
\hline & & $\lambda$ & $V(\phi) \sim$ \\
\hline$n=1$ & $q=1$ & $-1.7 \pm 2.1 i$ & $\left(\phi-\phi_{0}\right)^{-1}$ \\
& $q=2$ & $-1.2 \pm 2.1 i$ & $\left(\phi-\phi_{0}\right)^{-4}$ \\
& $q=2.5$ & $-1 \pm 2 i$ & $\left(\phi-\phi_{0}\right)^{-10}$ \\
\hline \multirow{2}{*}{$n=\frac{3}{2}$} & $q=1$ & $-1.7 \pm 1.5 i$ & $\left(\phi-\phi_{0}\right)^{-0.9}$ \\
& $q=2$ & $-1.2 \pm 1.5 i$ & $\left(\phi-\phi_{0}\right)^{-4}$ \\
& $q=2.5$ & $-1 \pm 1.4 i$ & $\left(\phi-\phi_{0}\right)^{-3.7}$ \\
\hline \multirow{3}{*}{$n=2$} & $q=1$ & $-1.7 \pm 1.1 i$ & $\left(\phi-\phi_{0}\right)^{-0.8}$ \\
& $q=2$ & $-1.2 \pm 1.2 i$ & $\left(\phi-\phi_{0}\right)^{-2}$ \\
& $q=2.5$ & $-1 \pm 1.1 i$ & $\left(\phi-\phi_{0}\right)^{-2.8}$ \\
\hline
\end{tabular}

where

$$
\begin{aligned}
V_{0} & =\frac{[6 n-(2 n-1) q] \rho_{\phi}^{(0)}}{6 n}\left[\frac{2 n}{3 n-q}\left(\frac{q \rho_{\phi}^{(0)} 2^{n-1}}{3 F_{0} n}\right)^{1 / 2 n}\right. \\
\left.\cdot\left(\frac{3 M_{p}^{2}}{\rho_{m}^{(0)}}\right)^{1 / 2}\right]^{2 n q /(q-3 n)} &
\end{aligned}
$$

Hence, as radiation epoch, we can find the linearized equation as

$$
\begin{aligned}
& \dot{u}_{1}(t)=0+p_{1}(t), \\
& \dot{p}_{1}(t)=3\left[\frac{q}{2}-\frac{3 n}{2 n-1}\right] u_{1}(t)+\left[q-\frac{9}{2}\right] p_{1}(t) .
\end{aligned}
$$

The eigenvalues of small oscillations around critical point are given by

$$
\lambda_{n, q}=f(n, q) \pm i g(n, q),
$$

where

$$
\begin{aligned}
& f(n, q)=-\frac{(9-2 q)}{4} \\
& g(n, q)=\left[\frac{144 n-\left(81-12 q+4 q^{2}\right)(2 n-1)}{16(2 n-1)}\right]^{1 / 2} .
\end{aligned}
$$

These eigenvalues for $0<q<3$ and different values of $n$ are brought at Table 2 (as was mentioned before, for $0<$ $q<3$, the ratio of scalar field to matter energy densities is an increasing function of time). It is shown that (from Table 2) for all values of $n=1,1.5,2$ and $q=1,2,2.5$ the eigenvalues $\lambda$ are $\lambda=\alpha \pm i \beta$ where $\alpha<0$ and $\beta \neq 0$; then all critical points are spiral and stable.

4.3. Scalar Field Dominated Epoch. We assume that the scalar field energy density is dominated and therefore we neglect the other components of the Universe. According to conservation equation for scalar field, (14), and the equation of state of scalar field, $p_{\phi}=(q / 3-1) \rho_{\phi}$, one finds $\gamma=q$; therefore the scalar field energy density is obtained as

$$
\rho_{\phi}=\rho_{\phi}^{(0)}\left(\frac{a_{0}}{a}\right)^{q}
$$




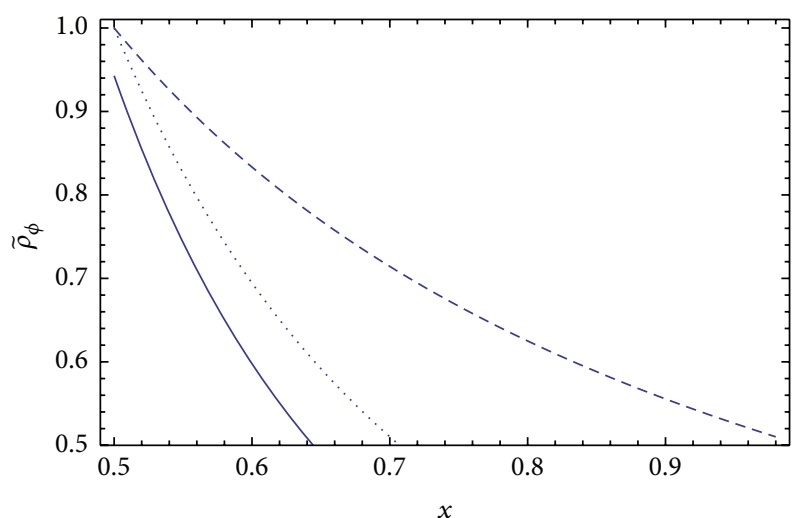

FIGURE 2: $\widetilde{\rho}_{\phi}:=\rho_{\phi} / \rho_{\phi}^{(0)}$ versus $x$ is plotted, where the dashed, dotted, and solid lines are denoted by $q=1,2,2.5$, respectively.

where $\rho_{\phi}^{(0)}$ is the value of scalar field energy density at $a=a_{0}$ (see Figure 2).

So, by substituting $q$ instead of $\gamma$ in (20) and integrating it for $n=1$, we have

$$
\phi-\phi_{0}=\sqrt{q M_{p}^{2}} \ln \left(\frac{a}{a_{0}}\right),
$$

where $\phi_{0}$ is the value of $\phi$ at $a=a_{0}$. So using (18) and (51) we have

$$
V(\phi)=\left[\frac{6-q}{6}\right] \rho_{\phi}^{(0)} e^{-\left(\sqrt{q} / M_{p}\right)\left(\phi-\phi_{0}\right)},
$$

and for $n \neq 1$ we obtain

$$
\begin{aligned}
& \phi-\phi_{0} \\
& =\frac{2 n}{q(n-1)}\left[\frac{q \rho_{\phi}^{(0)}}{6 F_{0} n}\right]^{1 / 2 n}\left(\frac{6 M_{p}^{2}}{\rho_{\phi}^{(0)}}\right)^{1 / 2}\left(\frac{a}{a_{0}}\right)^{q(n-1) / 2 n}, \\
& V(\phi)=V_{0}\left(\phi-\phi_{0}\right)^{2 n /(1-n)},
\end{aligned}
$$

where

$$
\begin{aligned}
V_{0} & =\frac{[6 n-(2 n-1) q] \rho_{\phi}^{(0)}}{6 n}\left[\frac{2 n}{q(n-1)}\left(\frac{q \rho_{\phi}^{(0)}}{6 F_{0} n}\right)^{1 / 2 n}\right. \\
& \left.\cdot\left(\frac{6 M_{p}^{2}}{\rho_{\phi}^{(0)}}\right)^{1 / 2}\right]^{2 n /(n-1)} .
\end{aligned}
$$

Also the linearized form of Einstein scalar field equation (with these potentials) is given by

$$
\begin{aligned}
& \dot{u}_{1}(t)=0+p_{1}(t), \\
& \dot{p}_{1}(t)=q\left[\frac{q}{2}-\frac{3 n}{2 n-1}\right] u_{1}(t)+\left[\frac{q}{2}-3\right] p_{1}(t) .
\end{aligned}
$$

The eigenvalues of small oscillations around critical point are given by

$$
\lambda_{n, q}=f(n, q) \pm i g(n, q)
$$

TABLE 3: The eigenvalues $\lambda$ for different values of $n$ and $q$, in scalar field dominated eppoch.

\begin{tabular}{cccc}
\hline & & $\lambda$ & $V(\phi) \sim$ \\
\hline \multirow{4}{*}{$n=1$} & $q=1$ & $-1.7 \pm 0.7 i$ & $e^{-\left(\left(\phi-\phi_{0}\right) / M_{p}\right)}$ \\
& $q=2$ & $-1.5 \pm 1.9$ & $e^{-\left(\sqrt{2}\left(\phi-\phi_{0}\right) / M_{p}\right)}$ \\
& $q=2.5$ & $-1.4 \pm 2.2 i$ & $e^{-\left(\sqrt{2.5}\left(\phi-\phi_{0}\right) / M_{p}\right)}$ \\
$n=\frac{3}{2}$ & $q=1$ & $-1.7 \pm 0.9$ & $\left(\phi-\phi_{0}\right)^{-6}$ \\
& $q=2$ & $-1.5 \pm 1.1 i$ & $\left(\phi-\phi_{0}\right)^{-6}$ \\
$n=2$ & $q=2.5$ & $-1.4 \pm 1.3 i$ & $\left(\phi-\phi_{0}\right)^{-6}$ \\
\hline \multirow{2}{*}{$n$} & $q=1$ & $-1.7 \pm 1.1$ & $\left(\phi-\phi_{0}\right)^{-4}$ \\
& $q=2$ & $-1.5 \pm 0.7 i$ & $\left(\phi-\phi_{0}\right)^{-4}$ \\
& $q=2.5$ & $-1.4 \pm 0.9 i$ & $\left(\phi-\phi_{0}\right)^{-4}$ \\
\hline
\end{tabular}

where

$$
\begin{aligned}
& f(n, q)=-\frac{8-q}{4} \\
& g(n, q)=\left[\frac{80 n q-\left[9 q^{2}+64\right][2 n-1]}{16(2 n-1)}\right]^{1 / 2} .
\end{aligned}
$$

These eigenvalues for $0<q<3$ and different $n$ are given at Table 3. It is clearly seen that all eigenvalues which are complex, such as $\lambda=\alpha \pm \beta i$ where $\alpha<0$ and $\beta \neq 0$, describe critical points which are spiral critical point and stable. For $n=1$ and $q=2$ the eigenvalues are $\lambda_{1}=0.4$ and $\lambda_{2}=-3.4$ so the critical point is a saddle point. For $n=3 / 2,2$ and $q=1$ the different eigenvalues are negative and stable.

Now we want to estimate the mass of the scalar field, $m_{\phi}$, in the scalar field dominated era. It is obvious that, by expanding the scalar field around the background field, one can suppose $m_{\phi}^{2}=d^{2} V(\phi) /\left.2 d \phi^{2}\right|_{\phi=\phi_{0}}$. Hence, for the potential function which was obtained for $n \neq 1$ in (54), one has

$$
m_{\phi}^{2}=\frac{d^{2} V(\phi)}{2 d \phi^{2}}=n V_{0} \frac{3 n-1}{(1-n)^{2}}\left(\phi-\phi_{0}\right)^{(5 n-3) /(1-n)} .
$$

By substituting (53) and (55) in the above equation, the mass of scalar field could be obtained as $m_{\phi}=3.5 \times$ $10^{-25}\left(a_{0} / a_{t}\right)^{0.1} \mathrm{~cm}^{-1}$. To get this value, we have taken $n=1.2$, $q=2.5, F_{0}=7.0, \rho_{\phi}^{(0)}=3.5 \times 10^{-25} \mathrm{~g} / \mathrm{cm}^{3}$, and $M_{p}=$ $2.5 \times 10^{-5} \mathrm{~g}$. It should be noted that this value is in good agreement with previous works [60].

\section{Conclusion and Discussion}

It is undoubtedly accepted that the scalar field mechanism attracts more attention in recent semicentury. For instance a wide range of problems and concepts such as hierarchy problem, steady state cosmology models, inflation, CDM, and dark energy by means of scalar field mechanism have been investigated. Amongst all different proposals of scalar field mechanism we have considered the noncanonical scenario, because of its advantages in solving coincidence and fine tuning problems. For such model we successfully have obtained a definite form of scalar field potential. It was shown that, for 
definite scalar field potential, in any related epoch, the scalar field solutions for an appropriate range of parameters are stable. In fact, it has indicated that, for example, in radiation and matter dominant eras, all eigenvalues of small oscillations around critical point are as $\lambda=\alpha+i \beta$, where $\alpha<0$ and $\beta \neq 0$. Also it should be noted that all critical points were spiral and stable. In addition, in scalar field epoch for $(n, q)=(1,2)$, the eigenvalues are $\lambda_{1}=0.4, \lambda_{2}=-3.4$; hence the critical point is a saddle point; for $(n, q)=(3 / 2,1)$ and $(2,1)$, the different eigenvalues are negative; therefore the critical point is stable. Also, for scalar field dominated era, by means of some wellknown values for this epoch, the mass of scalar field has been obtained as $m_{\phi}=3.5 \times 10^{-25}\left(a_{0} / a_{t}\right)^{0.1} \mathrm{~cm}^{-1}$.

From the results which are shown in the tables it is seen that for $n \neq 1$ in all epochs the potential has inverse power law form, and this indicates that the better candidate potential for explaining the evaluation of universe especially in inflation epoch has inverse power law form. Furthermore, our results show that for different form of noncanonical model we have different potential but all of them are inverse power law.

\section{Conflict of Interests}

The authors declare that there is no conflict of interests regarding the publication of this paper.

\section{Acknowledgment}

H. Sheikhahmadi would like to thank Iran's National Elites Foundation for financial support during this work.

\section{References}

[1] G. Efstathiou and J. R. Bond, "Cosmic confusion: degeneracies among cosmological parameters derived from measurements of microwave background anisotropies," Monthly Notices of the Royal Astronomical Society, vol. 304, no. 1, pp. 75-97, 1999.

[2] J. Dunkley, D. N. Spergel, E. Komatsu et al., "Five-year Wilkinson Microwave Anisotropy Probe (WMAP) observations: bayesian estimation of CMB polarization maps," The Astrophysical Journal, vol. 701, no. 2, pp. 1804-1813, 2009.

[3] A. G. Riess, A. V. Filippenko, P. Challis et al., "Observational evidence from supernovae for an accelerating universe and a cosmological constant," The Astronomical Journal, vol. 116, no. 3, pp. 1009-1038, 1998.

[4] S. Perlmutter, G. Aldering, G. Goldhaber et al., "Measurements of $\Omega$ and $\Lambda$ from 42 high-redshift supernovae," The Astrophysical Journal, vol. 517, no. 2, p. 565, 1999.

[5] D. J. Eisenstein and H. U. Wayne, "Baryonic features in the matter transfer function," Astrophysical Journal, vol. 496, no. 2, pp. 605-614, 1998.

[6] M. Shoji, D. Jeong, and E. Komatsu, "Extracting angular diameter distance and expansion rate of the universe from two-dimensional galaxy power spectrum at high redshifts: baryon acoustic oscillation fitting versus full modeling," The Astrophysical Journal, vol. 693, no. 2, article 1404, 2009.

[7] O. Farooq and B. Ratra, "Hubble parameter measurement constraints on the cosmological deceleration-acceleration transition redshift," The Astrophysical Journal Letters, vol. 766, no. 1, 2013.
[8] G. Chen, J. Gott III, and B. Ratra, "Non-gaussian error distribution of hubble constant measurements," Publications of the Astronomical Society of the Pacific, vol. 115, no. 813, pp. 12691279, 2003.

[9] M. Tegmark, M. A. Strauss, M. R. Blanton et al., "Cosmological parameters from SDSS and WMAP," Physical Review D, vol. 69, Article ID 103501, 2004.

[10] J. Sollerman, E. Mörtsell, T. M. Davis et al., "First-year sloan digital sky survey-II (SDSS-II) supernova results: constraints on nonstandard cosmological models," The Astrophysical Journal, vol. 703, no. 2, p. 1374, 2009.

[11] D. N. Spergel, L. Verde, H. V. Peiris et al., "First-year Wilkinson Microwave Anisotropy Probe (WMAP) observations: determination of cosmological parameters," The Astrophysical Journal Supplement Series, vol. 148, no. 1, p. 175, 2003.

[12] G. Hinshaw, J. L. Weiland, R. S. Hill et al., "Five-year Wilkinson microwave anisotropy probe observations: data processing, sky maps, and basic results," The Astrophysical Journal Supplement Series, vol. 180, no. 2, pp. 225-245, 2009.

[13] K. Bamba, S. Capozziello, S. Nojiri, and S. D. Odintsov, "Dark energy cosmology: the equivalent description via different theoretical models and cosmography tests," Astrophysics and Space Science, vol. 342, no. 1, pp. 155-228, 2012.

[14] P. A. R. Ade, N. Aghanim, C. Armitage-Caplan et al., "Planck 2013 results. IX. HFI spectral response," Astronomy \& Astrophysics, vol. 571, article A9, 2014.

[15] P. A. R. Ade, R. W. Aikin, D. Barkats et al., "Detection of Bmode polarization at degree angular scales by BICEP2," Physical Review Letters, vol. 112, no. 24, Article ID 241101, 25 pages, 2014.

[16] A. Einstein, "Kosmologische Betrachtungen zur allgemeinen Relativitätstheorie," Sitzungsberichte der Königlich Preussischen Akademie der Wissenschaften zu Berlin, vol. 142, 1917.

[17] H. Antoon Lorentz, The Prnciple of Relativity, Dover, New York, NY, USA, 1952.

[18] S. Weinberg, "The cosmological constant problem," Reviews of Modern Physics, vol. 61, no. 1, 1989.

[19] V. Sahni and A. A. Starobinsky, "The case for a positive cosmological $\Lambda$-term," International Journal of Modern Physics $D$, vol. 9, no. 4, pp. 373-443, 2000.

[20] P. J. Peebles and B. Ratra, "The cosmological constant and dark energy," Reviews of Modern Physics, vol. 75, no. 2, pp. 559-606, 2003.

[21] T. Padmanabhan, "Cosmological constant-the weight of the vacuum," Physics Reports, vol. 380, no. 5-6, pp. 235-320, 2003.

[22] P. J. Steinhardt, "Cosmological challenges for the 21st century," in Critical Problems in Physics, V. L. Fitch and D. R. Marlow, Eds., Printed University Press, Prinston, NJ, USA, 1997.

[23] D. Wands, "Extended gravity theories and the Einstein-Hilbert action," Classical and Quantum Gravity, vol. 11, no. 1, article 269, 1994.

[24] S. Nojiri and S. D. Odintsov, "Modified gravity as an alternative for $\Lambda$ CDM cosmology," Journal of Physics A: Mathematical and Theoretical, vol. 40, no. 25, article S17, pp. 6725-6732, 2007.

[25] A. Aghmohammadi, Kh. Saaidi, M. R. Abolhassani, and A. Vajdi, "Spherical symmetric solution in $f(R)$ model around charged black hole," International Journal of Theoretical Physics, vol. 49, no. 4, pp. 709-716, 2010.

[26] K. Saaidi, A. Vajdi, S. W. Rabiei, A. Aghamohammadi, and H. Sheikhahmadi, "Strong and weak gravitational field in $R+\mu^{4} / R$ gravity," Astrophysics and Space Science, vol. 337, no. 2, pp. 739745, 2012. 
[27] R. Ferraro and F. Fiorini, "Modified teleparallel gravity: inflation without an inflaton," Physical Review D, vol. 75, no. 8, Article ID 084031, 2007.

[28] R. Ferraro and F. Fiorini, "Born-Infeld gravity in Weitzenböck spacetime," Physical Review D, vol. 78, no. 12, Article ID 124019, 2008.

[29] K. Karami and A. Abdolmaleki, "Generalized second law of thermodynamics in $f(T)$ gravity," Journal of Cosmology and Astroparticle Physics, vol. 2012, no. 4, article 007, 2012.

[30] A. Aghamohammadi, "Holographic $f(T)$ gravity model," Astrophysics and Space Science, vol. 352, no. 1, pp. 1-5, 2014.

[31] H. Sheikhahmadi, A. Aghamohammadi, and K. Saaidi, "Noncommutative and commutative vacua effects in a scalar torsion scenario," Physics Letters B, vol. 749, pp. 231-235, 2015.

[32] S. Capozziello, V. F. Cardone, and V. Salzano, "Cosmography of $f(R)$ gravity," Physical Review D, vol. 78, no. 6, Article ID 063504, 2008.

[33] S. Capozziello and V. Salzano, "Cosmography and large scale structure by $f(R)$-gravity: new results," Advances in Astronomy, vol. 2009, Article ID 217420, 34 pages, 2009.

[34] M. Bouhmadi-López, S. Capozziello, and V. F. Cardone, "Cosmography of $f(R)$-brane cosmology," Physical Review D, vol. 82, no. 10, Article ID 103526, 2010.

[35] S. Capozziello, V. F. Cardone, H. Farajollahi, and A. Ravanpak, "Cosmography in $f(T)$ gravity," Physical Review D, vol. 84, no. 4, Article ID 043527, 2011.

[36] A. G. Cohen, D. B. Kaplan, and A. E. Nelson, "Effective field theory, black holes, and the cosmological constant," Physical Review Letters, vol. 82, no. 25, article 4971, 1999.

[37] B. Guberina, R. Horvat, and H. Nikolić, "Non-saturated holographic dark energy," Journal of Cosmology and Astroparticle Physics, vol. 2007, no. 1, article 012, 2007.

[38] L. Susskind, “The world as a hologram," Journal of Mathematical Physics, vol. 36, no. 11, article 6377, 1995.

[39] M. Li, "A model of holographic dark energy," Physics Letters B, vol. 603, no. 1-2, pp. 1-5, 2004.

[40] K. Enqvist and M. S. Sloth, "Possible connection between the location of the cutoff in the cosmic microwave background spectrum and the equation of state of dark energy," Physical Review Letters, vol. 93, no. 22, Article ID 221302, 2004.

[41] L. Hollenstein, M. Jaccard, M. Maggiore, and E. Mitsou, "Zeropoint quantum fluctuations in cosmology," Physical Review D, vol. 85, Article ID 124031, 2012.

[42] M. Maziashvili, "Space-time in light of Károlyházy uncertainty relation," International Journal of Modern Physics D, vol. 16, no. 9, pp. 1531-1539, 2007.

[43] M. Maziashvili, "Cosmological implications of Károlyházy uncertainty relation," Physics Letters B, vol. 652, no. 4, pp. 165168, 2007.

[44] H. Wei and R.-G. Cai, "A new model of agegraphic dark energy," Physics Letters B, vol. 660, no. 3, pp. 113-117, 2008.

[45] M. Maggiore, "Zero-point quantum fluctuations and dark energy," Physical Review D, vol. 83, no. 6, Article ID 063514, 2011.

[46] S. A. Fulling and L. Parker, "Renormalization in the theory of a quantized scalar field interacting with a robertson-walker spacetime," Annals of Physics, vol. 87, no. 1, pp. 176-204, 1974.

[47] H. Sheikhahmadi, A. Aghamohammadi, and Kh. Saaidi, "Vacuum dark energyin light of Planck 2013 in a de-Sitter like background," http://arxiv.org/abs/1407.0125.
[48] Y. B. Zeldovich and M. Yu. Khlopov, "On the concentration of relic magnetic monopoles in the universe," Physics Letters B, vol. 79, no. 3, pp. 239-241, 1978.

[49] J. P. Preskill, "Cosmological production of superheavy magnetic monopoles," Physical Review Letters, vol. 43, no. 19, article 1365, 1979.

[50] V. L. Ginzburg and L. D. Landau, "On the theory of superconductivity," Zhurnal Eksperimental'noi i Teoreticheskoi Fiziki, vol. 20, pp. 1064-1082, 1950.

[51] P. W. Higgs, "Broken symmetries and the masses of gauge bosons," Physical Review Letters, vol. 13, pp. 508-509, 1964.

[52] F. Englert and R. Brout, "Broken symmetry and the mass of gauge vector mesons," Physical Review Letters, vol. 13, pp. 321323, 1964.

[53] A. H. Guth, "Inflationary universe: a possible solution to the horizon and flatness problems," Physical Review D, vol. 23, no. 2, pp. 347-356, 1981.

[54] A. D. Linde, "A new inflationary universe scenario: a possible solution of the horizon, flatness, homogeneity, isotropy and primordial monopole problems," Physics Letters B, vol. 108, no. 6, pp. 389-393, 1982.

[55] T. Golanbari, A. Mohammadi, and K. Saaidi, "Brane inflation driven by noncanonical scalar field," Physical Review D, vol. 89, Article ID 103529, 2014.

[56] A. Aghamohammadi, A. Mohammadi, T. Golanbari, and K. Saaidi, "Hamilton-Jacobi formalism for tachyon inflation," Physical Review D: Particles, Fields, Gravitation and Cosmology, vol. 90, no. 8, Article ID 084028, 2014.

[57] C. H. Brans and R. H. Dicke, "Mach's principle and a relativistic theory of gravitation," Physical Review, vol. 124, no. 3, article 935, 1961.

[58] C. H. Brans, "Mach's principle and a relativistic theory of gravitation. II," Physical Review, vol. 125, no. 6, article 2194, 1962.

[59] P. J. E. Peebles and B. Ratra, "Cosmology with a time-variable cosmological constant," The Astrophysical Journal Letters, vol. 325, pp. L17-L20, 1988.

[60] B. Ratra and P. J. E. Peebles, "Cosmological consequences of a rolling homogeneous scalar field," Physical Review D, vol. 37, no. 12, pp. 3406-3427, 1988.

[61] C. Wetterich, "Cosmology and the fate of dilatation symmetry," Nuclear Physics, Section B, vol. 302, no. 4, pp. 668-696, 1988.

[62] T. G. Clemson and A. R. Liddle, "Observational constraints on thawing quintessence models," Monthly Notices of the Royal Astronomical Society, vol. 395, no. 3, pp. 1585-1590, 2009.

[63] R. R. Caldwell, "A phantom menace? Cosmological consequences of a dark energy component with super-negative equation of state," Physics Letters B, vol. 545, no. 1-2, pp. 23-29, 2002.

[64] R. R. Caldwell, M. Kamionkowski, and N. N. Weinberg, "Phantom energy: dark energy with $w<-1$ causes a cosmic doomsday," Physical Review Letters, vol. 91, no. 7, Article ID 071301, 2003.

[65] X.-M. Chen, Y. Gong, and E. N. Saridakis, "Phase-space analysis of interacting phantom cosmology," Journal of Cosmology and Astroparticle Physics, vol. 2009, no. 4, article 001, 2009.

[66] J. M. Cline, S. Jeon, and G. D. Moore, "The phantom menaced: constraints on low-energy effective ghosts," Physical Review D, vol. 70, no. 4, Article ID 043543, 2004.

[67] E. Elizalde, S. Nojiri, and S. D. Odinstov, "Late-time cosmology in a (phantom) scalar-tensor theory: dark energy and the 
cosmic speed-up," Physical Review D, vol. 70, no. 4, Article ID 043539, 20 pages, 2004.

[68] S. Nojiri, S. D. Odintsov, and S. Tsujikawa, "Properties of singularities in the (phantom) dark energy universe," Physical Review D, vol. 71, no. 6, Article ID 063004, 16 pages, 2005.

[69] A. Anisimov, E. Babichev, and A. Vikman, "B-inflation," Journal of Cosmology and Astroparticle Physicssissa, vol. 2005, no. 06, article 006, 2005.

[70] J. Khoury and A. Weltman, "Chameleon fields: awaiting surprises for tests of gravity in space," Physical Review Letters, vol. 93, no. 17, Article ID 171104, 2004.

[71] J. Khoury and A. Weltman, "Chameleon cosmology," Physical Review D, vol. 69, no. 4, Article ID 044026, 2004.

[72] D. F. Mota and J. D. Barrow, "Varying alpha in a more realistic universe," Physics Letters B, vol. 581, no. 3-4, pp. 141-146, 2004.

[73] Kh. Saaid, H. Sheikahmadi, and J. Afzali, "Chameleon mechanism with a new potential," Astrophysics and Space Science, vol. 333, no. 2, pp. 501-505, 2011.

[74] K. Saaidi, A. Mohammadi, and H. Sheikhahmadi, " $\gamma$ Parameter and solar system constraint in chameleon-Brans-Dicke theory," Physical Review D, vol. 83, no. 10, Article ID 104019, 2011.

[75] K. Saaidi, H. Sheikhahmadi, T. Golanbari, and S. W. Rabiei, "On the holographic dark energy in chameleon scalar-tensor cosmology," Astrophysics and Space Science, vol. 348, no. 1, pp. 233-240, 2013.

[76] T. Golanbari, A. Mohammadi, and K. Saaidi, "QCD phase transition with a power law chameleon scalar field in the bulk," International Journal of Modern Physics A, vol. 29, no. 7, Article ID 1450033, 2014.

[77] A. Aghamohammadi, K. Saaidi, A. Mohammadi, H. Sheikhahmadi, T. Golanbari, and S. W. Rabiei, "Effect of an external interaction mechanism in solving agegraphic dark energy problems," Astrophysics and Space Science, vol. 345, no. 1, pp. 1724, 2013.

[78] S. M. Carroll, "Quintessence and the rest of the world: suppressing long-range interactions," Physical Review Letters, vol. 81, no. 15, pp. 3067-3070, 1998.

[79] C. Armendariz-Picon, V. F. Mukhanov, and P. J. Steinhardt, "Dynamical solution to the problem of a small cosmological constant and late-time cosmic acceleration," Physical Review Letters, vol. 85, no. 21, pp. 4438-4441, 2000.

[80] T. Chiba, T. Okabe, and M. Yamaguchi, "Kinetically driven quintessence," Physical Review D, vol. 62, no. 2, Article ID 023511, 2000.

[81] C. Armendariz-Picon, V. F. Mukhanov, and P. J. Steinhardt, "Essentials of $k$-essence," Physical Review D, vol. 63, no. 10, Article ID 103510, 2001.

[82] M. Alishahiha, E. Silverstein, and D. Tong, "DBI in the sky: nonGaussianity from inflation with a speed limit," Physical Review D, vol. 70, no. 12, Article ID 123505, 15 pages, 2004.

[83] A. Sen, "Tachyon matter," Journal of High Energy Physics, vol. 2002, no. 7, p. 65, 2002. 

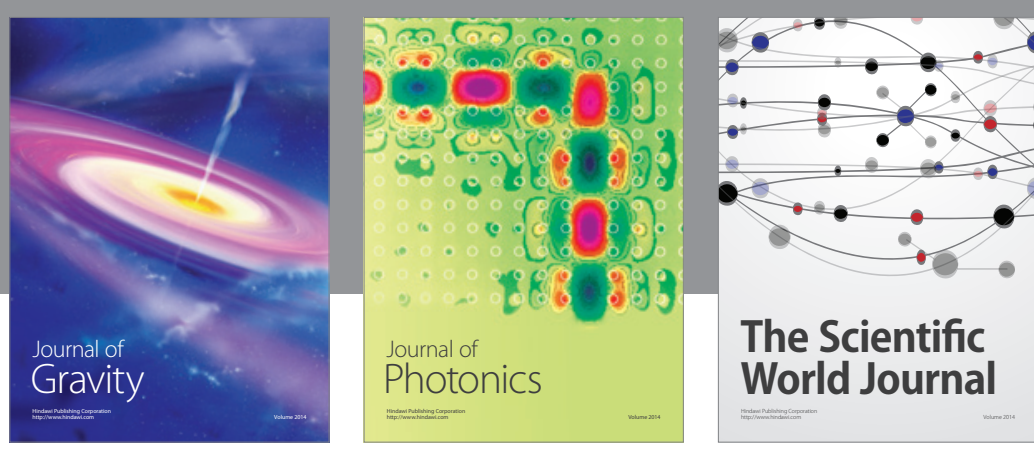

The Scientific World Journal
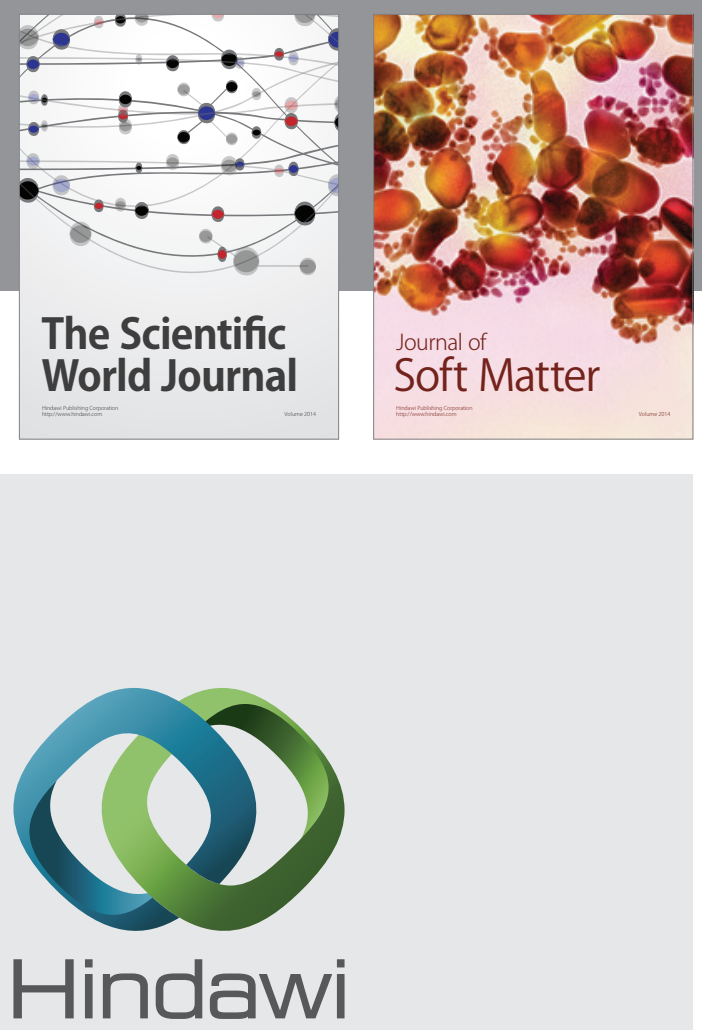

Submit your manuscripts at

http://www.hindawi.com

nternational Journal of

Statistical Mechanics
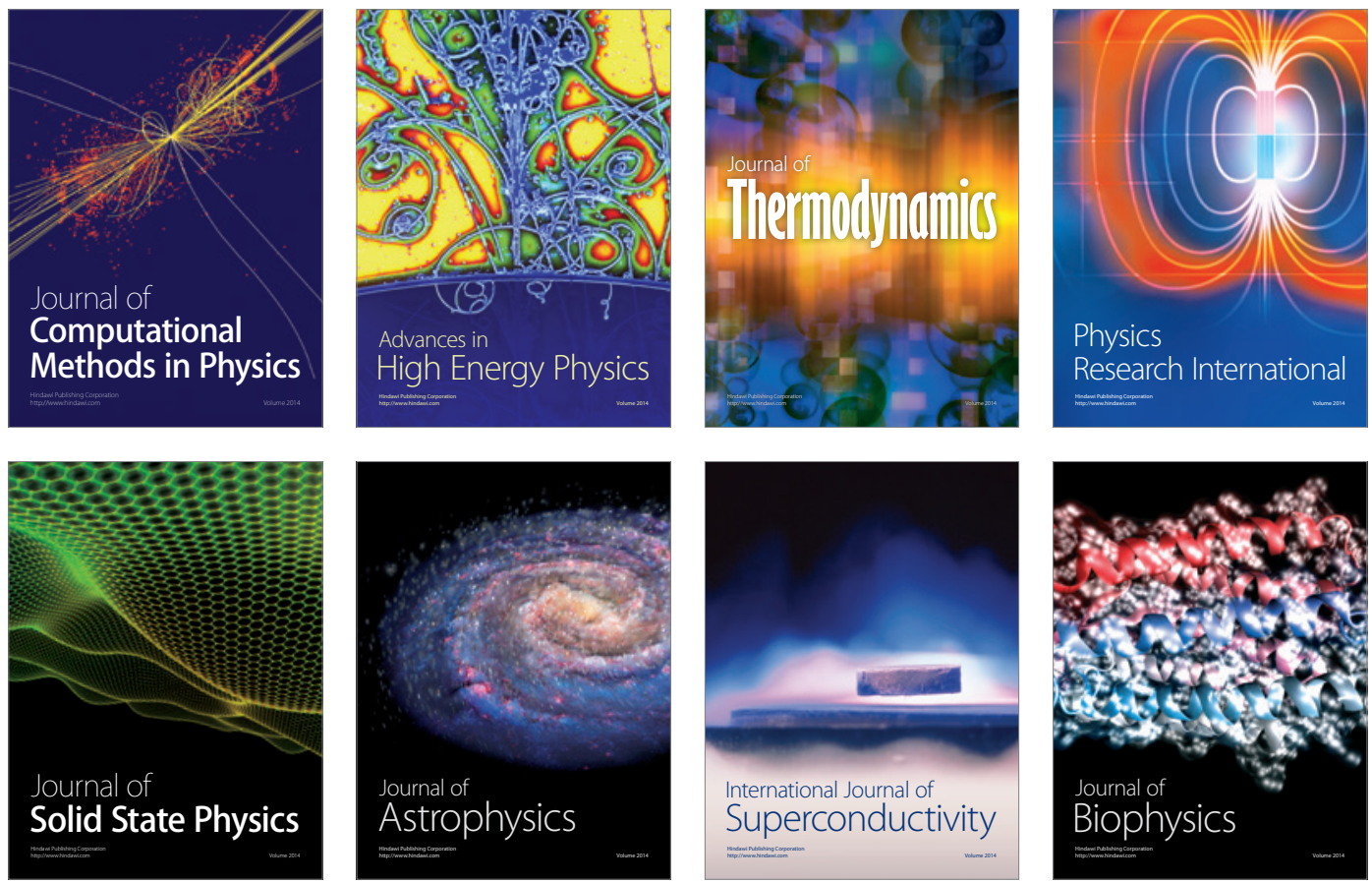
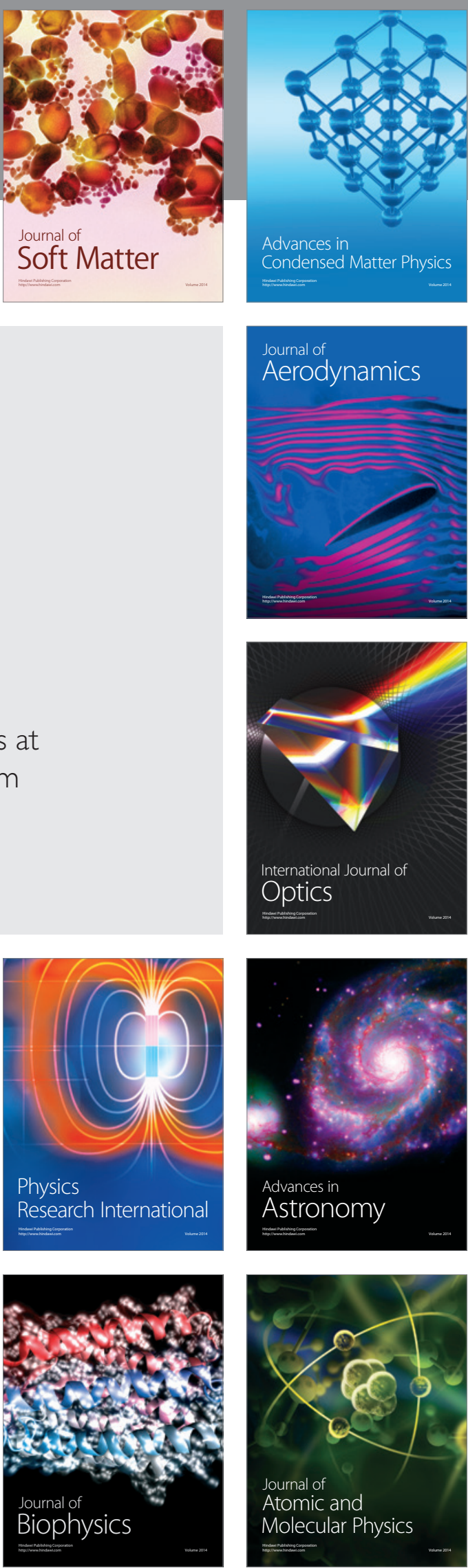